\title{
ORIGINAL ARTICLE \\ Selection of pairings reaching evenly across the data (SPREAD): A simple algorithm to design maximally informative fully crossed mating experiments
}

\author{
K Zimmerman ${ }^{1}$, D Levitis ${ }^{2}$, E Addicott ${ }^{1}$ and A Pringle ${ }^{3,4}$ \\ We present a novel algorithm for the design of crossing experiments. The algorithm identifies a set of individuals (a "crossing- \\ set') from a larger pool of potential crossing-sets by maximizing the diversity of traits of interest, for example, maximizing the \\ range of genetic and geographic distances between individuals included in the crossing-set. To calculate diversity, we use the \\ mean nearest neighbor distance of crosses plotted in trait space. We implement our algorithm on a real dataset of Neurospora \\ crassa strains, using the genetic and geographic distances between potential crosses as a two-dimensional trait space. \\ In simulated mating experiments, crossing-sets selected by our algorithm provide better estimates of underlying parameter \\ values than randomly chosen crossing-sets.
}

Heredity (2016) 116, 182-189; doi:10.1038/hdy.2015.88; published online 30 September 2015

\section{INTRODUCTION}

Researchers planning mating experiments are faced with a critical design choice- - deciding how many pairs and which pairs of individuals to mate. The number of crosses in a mating experiment can influence the statistical estimates of genetic effects and combining abilities (Jui and Lefkovitch, 1992). The selection of pairs to use in a mating experiment also affects the outcome of the experiment. For example, if the goal of a mating experiment is to understand the genetic basis of a trait, as in quantitative trait locus analysis, then parents should carefully be chosen to maximize the genetic diversity among offspring and increase the likelihood of detecting quantitative trait loci (Crepieux et al., 2004). The increasing accessibility of population genetic and genomic datasets offer genetic data on more individuals than can reasonably be used in most experiments (Cushman, 2014). This poses a methodological problem: how to choose a subsample of mating pairs that best reflects the range of cross characteristics (in two or more dimensions of genetic, geographic or ecological space) of the complete set of all available pairs.

One solution is to select a subsample that recapitulates the characteristics of the larger set and preserves underlying relationships between the variables used to define a trait space. The representative subsample might mimic the broad distribution of crosses in the larger set, in other words, it attempts to maintain the shape, clumps and so on of the larger set. Other subsampling methods include choosing samples by eye or randomly, but these methods may truncate the trait space by omitting outliers or disproportionately drawing from the dense center of a distribution. Omissions in sampling may hinder a complete understanding of how response variables, for example, reproduction, vary across the trait space of all possible crosses. Furthermore, predicting response variables outside the range of explanatory variables used in an experiment involves extreme value methods, which can increase the error associated with predictions, unless limiting assumptions are made (Pauli and Coles, 2001).

In breeding and mating studies, fully crossed matings between all possible pairs are desirable to determine the combining ability and genetic and maternal effects (Griffing, 1956; Zhu and Weir, 1996). But a directed subsampling of all potential crosses to achieve some other aim, for example, to maximize genetic diversity within the experiment, may result in a set of target individuals that cannot be fully crossed. A method is required to subsample and design matings to satisfy both aims, for example, to maximize genetic diversity and to fully cross all included individuals.

Algorithms for maximizing combinatorial diversity have been extensively developed in the context of generating diverse molecular libraries for drug screening (Martin and Critchlow, 1999). In these algorithms, the metric of diversity is based on 'redundancy' and 'coverage' (Martin and Critchlow, 1999). Redundancy is the overlapping or clumping of points in space, while coverage is the spread of points across the space. An ideal diversity metric would minimize redundancy while maximizing coverage. The algorithms used in chemical combinatorial analysis focus on maximizing the diversity of a subset of molecules from a larger set by step-wise analysis of differences between additional compounds added to a set (Holliday et al., 1995). These algorithms cannot be directly applied to our problem because they do not require selection of fully crossed sets. However, we use their definitions of ideal set diversity to derive our own measure of diversity that can be applied to fully crossed sets.

Calculating the mean of the nearest neighbor distances (NND) of points representing a full factorial set of crosses plotted based on their underlying parameters (for example, genetic, geographic or ecological

${ }^{1}$ Department of Organismic and Evolutionary Biology, Harvard University, Cambridge, MA, USA; ${ }^{2}$ Bates College, Department of Biology, Lewiston, ME, USA; ${ }^{3}$ Department of Botany, University of Wisconsin, Madison, WI, USA and ${ }^{4}$ Department of Bacteriology, University of Wisconsin, Madison, WI, USA

Correspondence: K Zimmerman, Department of Organismic and Evolutionary Biology, Harvard University, 16 Divinity Avenue, Biolabs 2112, Cambridge, MA 02138, USA.

E-mail: kzimmerman@fas.harvard.edu

Received 30 January 2015; revised 11 August 2015; accepted 13 August 2015; published online 30 September 2015 
distance) will give a measure of the evenness or 'non-redundancy' of the points. The mean NND is often used to determine whether a particular set of plotted points is randomly distributed or not (Clark and Evans, 1954). A set of plotted points that are clumped will result in a smaller value of the mean NND than a sample with the same number of more evenly and broadly distributed points. The maximum mean NND (MMNND) will occur when points are spread as evenly as possible and the 'coverage' of space is maximal (Wang and Cumming, 2011). Thus, identifying a set of crosses with the MMNND from a large random sample of many potential sets of crosses ('crossing-sets') will return a crossing-set that is both broad and even with respect to underlying trait values as compared with a randomly sampled crossing-set.

We introduce a simple algorithmic sampling method for choosing crossing-sets; we name the algorithm SPREAD (Selection of Pairings Reaching Evenly Across the Data). SPREAD is based on selecting the single crossing-set with the MMNND from among a large random sample of potential crossing-sets plotted on two-dimensional trait space. We use our algorithm to select a crossing-set from a genotyped collection of geographically widespread wild strains of the filamentous fungus, Neurospora crassa. Strains of this fungus have one of two mating types, denoted mat-A or mat-a. The two parents in a cross must have different mating types to mate. Recently, 24 strains of each mating type were genotyped using RNAseq (Ellison et al., 2011). The genotyped strains were collected from diverse locations, allowing us to assign both genetic (the number of different single nucleotide polymorphisms) and geographic (the distance between collection sites) distance values to each of the 576 potential crosses. Using this dataset as our example, we implemented the SPREAD algorithm and tested its effectiveness when the true MMNND is not easily calculable. Finally, we compare the ability of SPREAD selected and randomly sampled crossing-sets to estimate known parameter values that relate genetic and geographic distances to reproductive output for the entire set of all potential crosses.

\section{METHODS}

\section{General description of the SPREAD algorithm}

Define $\mathbb{X}$ and $\mathbb{Y}$ as the set of available strains or individuals of each mating type or sex ('type') $x$ and $y$, respectively, and $s_{x} \times s_{y}$ as the feasible number of crosses that can be completed in an experiment. The variables $s_{x}$ and $s_{y}$ are the number of strains selected for the experiment and are less than $|\mathbb{X}|$ and $|\mathbb{Y}|$, respectively. Draw a large number, $h$, of random samples containing $s_{x}$ and $s_{y}$ strains of each type from all possible sets of strains $\left(\begin{array}{c}\mathbb{X} \\ s_{x}\end{array}\right)$ and $\left(\begin{array}{c}\mathbb{Y} \\ s_{y}\end{array}\right)$. For each of the $h$ samples, plot crosses based on values associated with the crosses (for example, number of differing single nucleotide polymorphisms vs geographic distance), and then calculate the mean of the NNDs of all plotted crosses. Generate a list of $h$ mean NNDs. Finally, use the maximum value from the list because it corresponds to the set of $s_{x} \times s_{y}$ strains that most broadly and evenly represents the parameter of interest. A formal mathematical description of this algorithm is presented in the Supplementary Material (Supplementary File 1).

\section{A worked example using SPREAD}

We used a previously published population genomics dataset consisting of single nucleotide polymorphisms from transcriptomes of geographically diverse wild isolates of the fungus N. crassa to test our method (Ellison et al., 2011). We started with the set of all pairwise combinations of strains and then filtered to include only mating type compatible pairs. We calculated the genetic distances between compatible pairs by counting the number of different single nucleotide polymorphisms (SNPs) between each pair and calculated geographic distances using the great-circle distance between strain locales. The genetic and geographic distance values for each pair were used to map all the crosses on genetic and geographic distance axes. This is the 'original distribution' of crosses.

We randomly sampled $h=1000$ lists of $s_{A}=s_{a}=12$ strains of each mating type from the set of all $\left(\begin{array}{c}\mathbb{A} \\ s_{a}\end{array}\right)$ and $\left(\begin{array}{c}\mathbf{a} \\ s_{a}\end{array}\right)$ strains without replacement, where $\mathbb{A}$ and $\mathbf{a}$ are the sets of strains available for each mating type (analogous to $\mathbb{X}$ and $\mathbb{Y}$ in the general description); in this case $|\mathbb{A}|=|\mathbf{a}|=24$. We computed all possible pairwise mating combinations for each of the 1000 random samples of $s_{A}=s_{a}=12$ strain lists, resulting in 1000 crossing-sets each containing 144 crosses. We then plotted each crossing-set on geographic vs genetic distance space and computed the mean NNDs using Euclidean distance calculations. The crossing-set with the MMNND of all 1000 crossing-sets was selected. In this worked example, selecting from a random sample of 1000 crossing-setsand not selecting from all possible crossing-sets-is necessary because the total number of possible crossing-sets in this case is $\left(\begin{array}{l}24 \\ 12\end{array}\right)^{2}=7.31 \times 10^{12}$. Computing NNDs for all possible crossing-sets is computationally prohibitive and, as shown below, unnecessary.

We implemented our algorithm and additional analyses in the $\mathrm{R}$ programming language ( $\mathrm{R}$ Core Team, 2014). $\mathrm{R}$ code for the implementation of the SPREAD algorithm on crossing-sets with two traits and two sexes is available in the Supplementary Information (Supplementary File 2) and online at http://dx.doi. org $/ 10.6084 / \mathrm{m} 9$.figshare.1180165. The following $\mathrm{R}$ packages were used in this analysis: plyr (Wickham, 2011), reshape2 (Wickham, 2007), ggplot2 (Wickham, 2009), spatstat (Baddeley and Turner, 2005), foreach (Weston and Analytics, 2014) and glmmADMB (Fournier et al., 2012; Skaug et al., 2013).

\section{Evaluating SPREAD's approximation of the true MMNND}

The true MMNND can only be determined if all possible crossing-sets for a given $s_{A}$ and $s_{a}$ are evaluated. Therefore, calculating the true MMNND may not be possible, even with high performance computing resources. For example, if $\mathrm{M}=300$ and $\mathrm{F}=300$ and a crossing-set is desired with 20 individuals of each type, then the total number of possible crossing-sets would be $\left(\begin{array}{c}300 \\ 20\end{array}\right)^{2}=5.6 \times 10^{61}$. Using a random sample of all available crossing-sets to estimate the MMNND is more practical, especially if the estimated MMNND approximates the true MMNND.

We implemented the algorithm as described above for the N. crassa dataset, except we varied crossing-set size by implementing SPREAD for $s_{A}=s_{a}=2,3,4$, $\ldots, 22$. To simplify the process, we used crossing-sets where $s_{A}=s_{a}$, but this is not a requirement of the SPREAD algorithm. We used five different $h$ values $(1$, $10,100,1000$ and 10000) to compare the effects of $h$ size on the MMNND values returned from SPREAD. We included an $h$ value of 1 to simulate the distribution of MNND values of crossing-sets generated by simple random sampling (SRS). We repeated this process 1000 times to obtain bootstrapped distributions of MMNND values for the different crossing-set sizes and $h$ values.

Comparing model fits of SPREAD- and SRS-generated crossing-sets Using SPREAD to design fully crossed mating experiments may be more effective than selecting crossing-sets at random because broad and even sampling will provide greater statistical power to understand how dependent variables vary based on cross characteristics (for example, how reproductive success depends on the genetic or geographic distances between parents). To evaluate this hypothesis empirically, we created a simulated dataset of cross outcomes (that is, reproduction) and modeled relationships between reproduction and the characteristics of crosses in crossing-sets generated from SPREAD vs those generated by simple random sampling. Simulated experimental data take the form of total ascospore counts (the sexually produced spores of the fungus).

First, we generated simulated data for all possible crosses of the entire crossing-set of 24 mat-A $\times 24$ mat-a strains, using a generalized linear model fitted to unpublished empirical data from a diallel cross of 11 mat-a and 10 mat-A N. crassa strains. This model was evaluated using the glmmADMB package (Fournier et al., 2012; Skaug et al., 2013) as Total Ascospore Count = Genetic Distance+Geographic Distance+(Genetic Distance $)^{2}+($ Geographic Distance $)^{2}+$ Genetic Distance: Geographic Distance. The response variable of Total Ascospore Count was modeled with a negative binomial distribution using 
a $\log$ link function. Genetic and geographic distance values were mean-centered to prevent autocorrelation between linear and quadratic parameters. We did not include all possible interaction terms because we wanted to restrict the model to only linear and quadratic terms. Our choice is based on the biological hypothesis that there is a single optimum of reproductive output, intermediate between inbreeding and outbreeding depression (Lynch, 1991).

Second, we simulated four experimental replicates for each possible cross by drawing from a negative binomial distribution with a mean derived from the predicted experimental values and the negative binomial dispersion parameter derived from the empirical data model (Supplementary File 3). The model parameter values of the entire crossing population were determined by evaluating the model described above with the complete simulated data set of all crosses. The model parameter values from the entire population model will be referred to as 'population' parameter values, while parameter values generated from subsampled crossing-set models will be referred to as 'sample' parameter values.

Using the complete set of simulated experimental data, we computed data sets for 1000 different crossing-sets generated with either SPREAD or SRS. The algorithm parameter values for the SPREAD-generated crossing-sets were $s_{A}=s_{a}=12$ and $h=1000$. We chose $s_{A}=s_{a}=12$ to test the edge case of a maximally complex sample space (the largest number of possible crossing-set permutations occurs when $s_{A}=s_{a}=12$ ). Crossing-sets chosen by SRS were of the same size. Model fits were computed for each crossing-set using the model described above. Parameter values and standard errors of the parameter values were recorded for each of the 1000 SPREAD- or SRS-generated crossing-sets.

We compared the bias, precision and accuracy of sample parameter values from model fits of crossing-sets generated with SPREAD or SRS. To compute bias, we calculated the difference between the mean of sample parameter values from the 1000 model fits and the population parameter values. To assess precision, we computed the variance of sample parameter values from the 1000 model fits. To assess accuracy, we calculated mean squared error of sample parameter values as $\frac{1}{n} \sum_{i=1}^{n}\left(\hat{\theta}_{i}-\theta\right)^{2}$, where $n=1000, \hat{\theta}_{i}=$ the sample parameter value of simulation $i$ and $\theta=$ the population parameter value. We recorded the MNND values of all SPREAD- and SRS-generated crossing-sets used in this comparison to determine the relationship between MNND of crossing-sets and the ability of those crossing-sets to estimate population parameter values.

\section{RESULTS}

\section{The worked example}

We used SPREAD on the $N$. crassa dataset described above to select a crossing-set with 12 mat-A and 12 mat-a strains. A graphical assessment of the chosen crossing-set plotted on geographic and genetic distance axes shows that our method produces a crossing-set that broadly and evenly represents all potential crosses (Figure 1).

\section{Implementing SPREAD without knowing the true MMNND}

Although the goal of SPREAD is to find a crossing-set with a high MNND value, finding the true maximum MNND is not necessary for most experimental purposes. Rather, the number of randomly selected potential crossing-sets considered $(h)$ should balance the desirability of finding a set with a high MNND with computational convenience. Plotting distributions of MMNND values for the five $h$ values shows that $h$ values beyond 1000 deliver sharply diminishing marginal returns for all crossing-sets with more than 16 crosses (Figure 2). As the crossing-set size increases, MMNND values decrease because with more crosses, the average distance between crosses decreases. Furthermore, the range of the MMNND estimates decreases as $h$ increases, mainly driven by an increase of the lower bound while the upper bound remains relatively unchanged.

\section{Comparing SPREAD to SRS}

We tested the ability of SPREAD to generate crossing-sets that will more accurately predict the parameter values one might find if all potential matings of an available breeding population were used in an experiment. Repeated simulations yielded distributions of parameter estimates for each model parameter using each method (SPREAD or SRS) (Figure 3). For both SPREAD and SRS, the peaks of these distributions of estimates do not perfectly align with the underlying population parameter values (Figure 3a). For three of the six model parameters, the mean parameter value from SPREAD-generated crossing-sets is less biased than the mean parameter value from SRS-generated crossing-sets (Table 1). However, the distributions of all six sample parameter values from SPREAD-generated crossing-sets have smaller variances and mean squared errors than those from SRSgenerated crossing-sets (Table 1). The standard errors of parameter values from SPREAD-based models are small compared with those from SRS-based models (Figure $3 \mathrm{~b}$ and Table 2), increasing the probability of closely approximating the population parameter values.
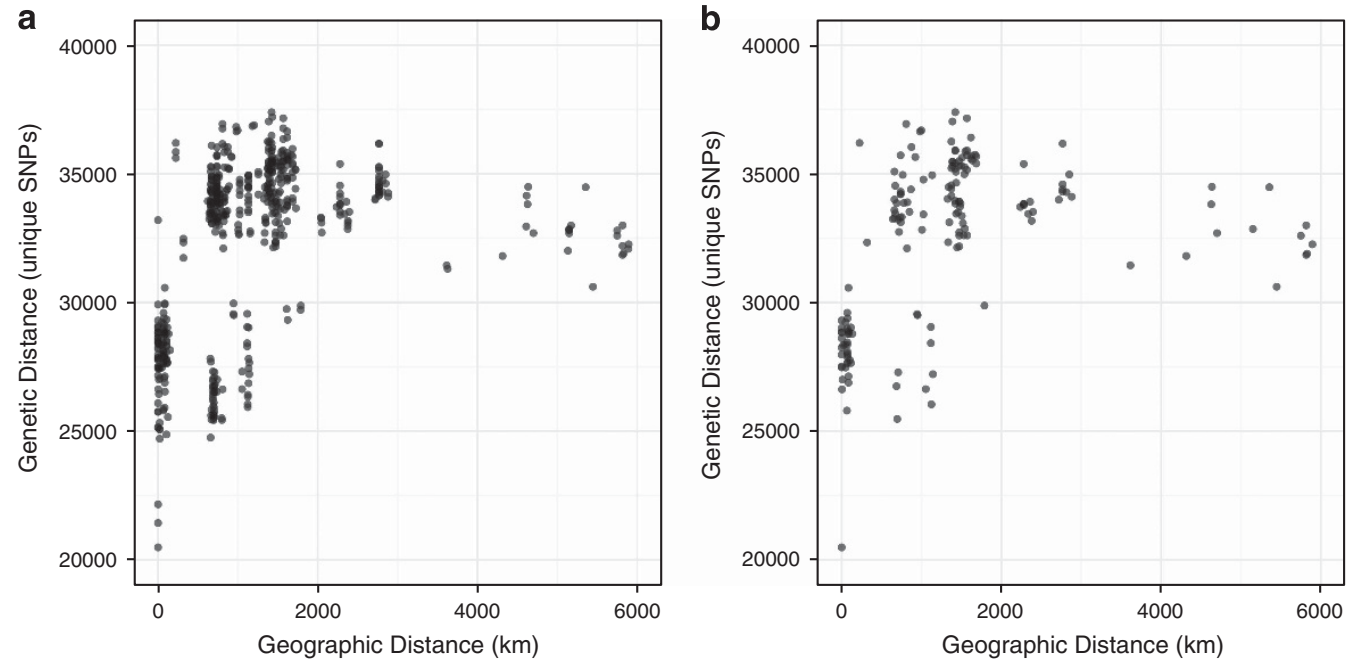

Figure 1 Example implementation of SPREAD. Panel (a) shows all possible crosses between 24 mat-A and 24 mat-a $N$. crassa strains plotted based on genetic and geographic distance between the parents in a cross; panel (b) shows the set of 12 mat-A $\times 12$ mat-a $\mathrm{N}$. crassa strains returned from an example run of the SPREAD algorithm (with $h=1000$ ). The SPREAD selected set can vary between runs of the algorithm. Crosses are plotted as semitransparent dots and darker colors mark overlapping crosses. 

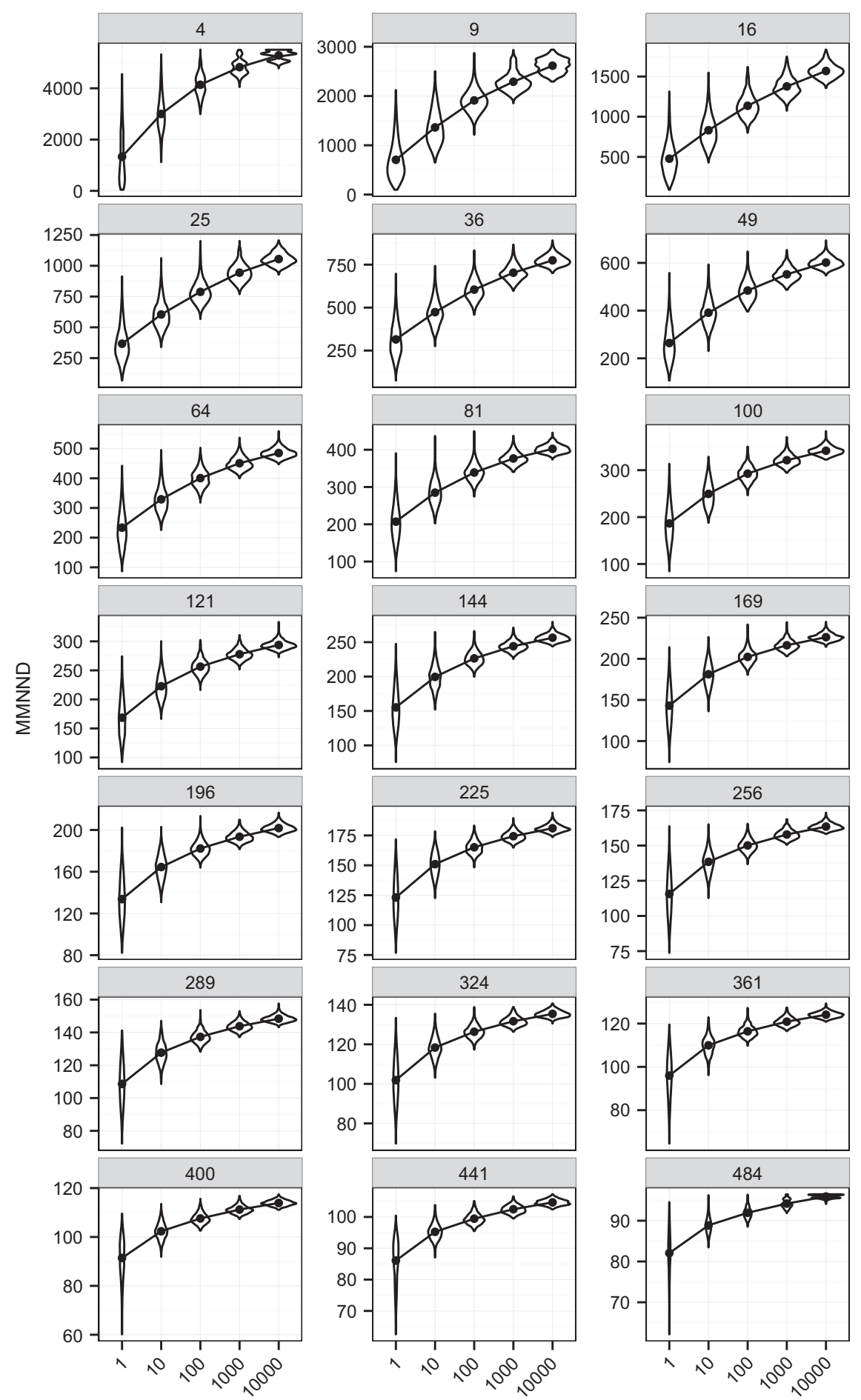

$h$ value

Figure 2 Effect of crossing-set size and $h$ value (the number of random crossing-sets from which the crossing-set is selected) on distributions of MMNND values of selected crossing-sets. Each facet is labeled with the size of the crossing-set. Violin plots show the entire distributions of MMNND values. Points and lines show how the mean of the distributions changes with increasing $h$ values.

\section{MNND vs parameter estimates}

Broadly and evenly distributed explanatory variables increase the accuracy and precision of predictions based on those variables. The MNND is a measure of the broadness and evenness of points in space, and so it should be negatively correlated with measures of inaccuracy or error. Indeed, we found strong negative relationships between the MNND of crossing-sets and both the deviation from population parameter values (Figure $4 \mathrm{a}$ ) and the standard errors of sample parameters (Figure 4b). Within SRS-generated crossingsets, those sets with higher MNND values also recapitulated the population parameter values better than crossing-sets with lower MNND values. 
a
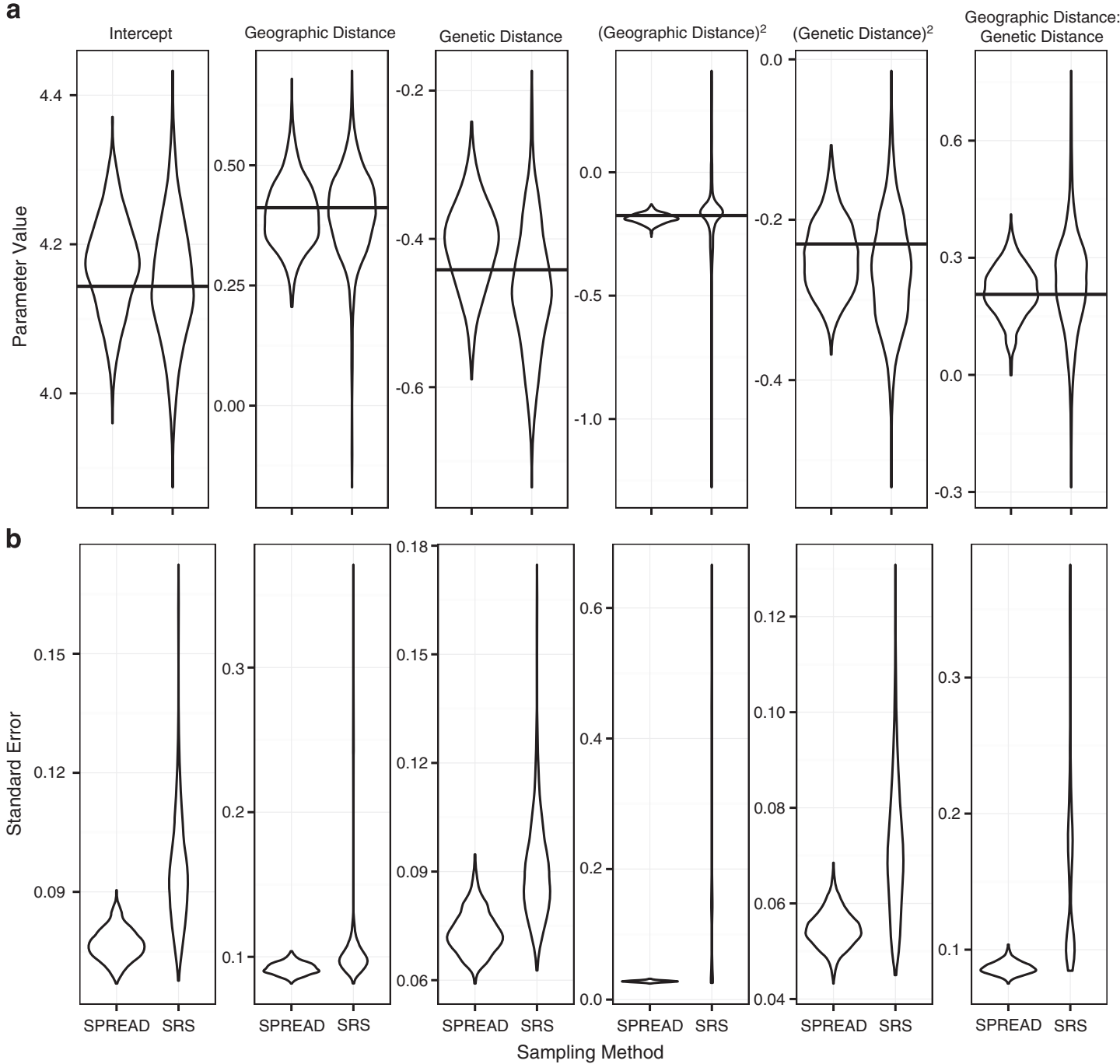

Figure 3 Comparisons of sample parameter values and standard errors for 1000 crossing-sets generated either with SPREAD or SRS. The SPREAD specifications used are $s_{A}=s_{a}=12$ and $h=1000$. (a) Violin plots showing the distribution of sample parameter values for each parameter in the model. Horizontal lines indicate the population parameter values of the entire simulated $A=a=24$ dataset. (b) Violin plots showing the distributions of standard errors of parameter values for each parameter in the model.

Table 1 Comparison of bias, variance and mean squared error of sample parameter values from model fits of crossing-sets generated with SPREAD or SRS

\begin{tabular}{|c|c|c|c|c|c|c|}
\hline \multirow[t]{2}{*}{ Model parameter } & \multicolumn{2}{|c|}{ Bias } & \multicolumn{2}{|c|}{ Variance } & \multicolumn{2}{|c|}{ Mean squared error } \\
\hline & SPREAD & SRS & SPREAD & SRS & SPREAD & SRS \\
\hline Intercept & 2.891E-02 & $6.454 \mathrm{E}-03$ & 4.110E-03 & $6.889 \mathrm{E}-03$ & $4.942 \mathrm{E}-03$ & $6.924 \mathrm{E}-03$ \\
\hline Genetic dist. & 3.374E-02 & $-2.646 \mathrm{E}-02$ & $3.356 \mathrm{E}-03$ & 7.176E-03 & 4.491E-03 & $7.869 \mathrm{E}-03$ \\
\hline Geographic dist. & $-1.140 \mathrm{E}-02$ & $-1.270 \mathrm{E}-02$ & 4.833E-03 & 8.842E-03 & $4.958 \mathrm{E}-03$ & 8.995E-03 \\
\hline$(\text { Genetic dist. })^{2}$ & $-1.561 \mathrm{E}-02$ & $-3.385 E-02$ & 2.022E-03 & 4.558E-03 & $2.263 \mathrm{E}-03$ & 5.700E-03 \\
\hline$(\text { Geographic dist. })^{2}$ & $-1.170 \mathrm{E}-02$ & $-1.770 \mathrm{E}-02$ & 3.758E-04 & 1.973E-02 & $5.122 \mathrm{E}-04$ & 2.003E-02 \\
\hline Geographic:Genetic dist. & 7.791E-03 & 3.189E-02 & 4.226E-03 & 1.740E-02 & $4.283 \mathrm{E}-03$ & $1.840 \mathrm{E}-02$ \\
\hline
\end{tabular}

Abbreviations: Dist., distance; SPREAD, selection of pairings reaching evenly across the data; SRS, simple random sampling. Lesser values (SPREAD vs SRS) are shown in bold.

\section{DISCUSSION}

SPREAD is an easily implemented algorithm designed to identify maximally informative, full factorial crossing-sets for use in mating experiments. SPREAD increases the diversity inherent in a crossing-set, for example, the genetic and geographic distances among crosses compared with a randomly sampled crossing-set. SPREAD requires two input parameters chosen by the user: the dimensions, $s_{x} \times s_{y}$, of the desired crossing-set and the number of randomly 
Table 2 Summary statistics for the distribution of standard errors from 1000 model fits using either SPREAD- or SRS-generated crossing-sets

\begin{tabular}{|c|c|c|c|c|}
\hline \multirow[t]{2}{*}{ Model parameter } & \multicolumn{2}{|c|}{ Mean of std. errors } & \multicolumn{2}{|c|}{ Variance of std. errors } \\
\hline & SPREAD & SRS & SPREAD & SRS \\
\hline Intercept & 0.0768 & 0.0944 & $1.60 \mathrm{E}-05$ & $1.72 \mathrm{E}-04$ \\
\hline Genetic distance & 0.0735 & 0.0904 & 3.59E-05 & 1.94E-04 \\
\hline Geographic distance & 0.0919 & 0.1093 & $1.66 \mathrm{E}-05$ & 1.60E-03 \\
\hline$(\text { Genetic distance })^{2}$ & 0.0547 & 0.0709 & $1.48 \mathrm{E}-05$ & $1.85 \mathrm{E}-04$ \\
\hline$(\text { Geographic distance })^{2}$ & 0.0280 & 0.1079 & $1.79 \mathrm{E}-06$ & 1.19E-02 \\
\hline Genetic Dist.:Geographic Dist. & 0.0867 & 0.1466 & $2.16 \mathrm{E}-05$ & 2.87E-03 \\
\hline
\end{tabular}

Abbreviations: Dist., distance; SPREAD, selection of pairings reaching evenly across

the data; SRS, simple random sampling. Lesser values (SPREAD vs SRS) are shown in bold. generated crossing-sets, $h$, from which the crossing-set with the MMNND is selected. The code for SPREAD was designed for two dimensional trait data. If potential crosses are characterized by more than two target traits, and the traits are not completely independent, principal components analysis can be used before implementing SPREAD as is to determine which two traits explain most of the trait variance (King and Jackson, 1999). Alternatively, the SPREAD code could be modified to calculate NNDs in multi-dimensional space.

In our worked example, we successfully used SPREAD to select a crossing-set of 12 mat-A $\times 12$ mat-a $N$. crassa strains. When these crosses are plotted in genetic vs geographic distance space, it is evident that the selected set fulfills the desired criteria of evenly and completely covering the range of the larger set (Figure 1). Using the MMNND as the diversity metric favors crosses that are at the extremes of the traitspace. The inclusion of crosses with extreme trait distances in an
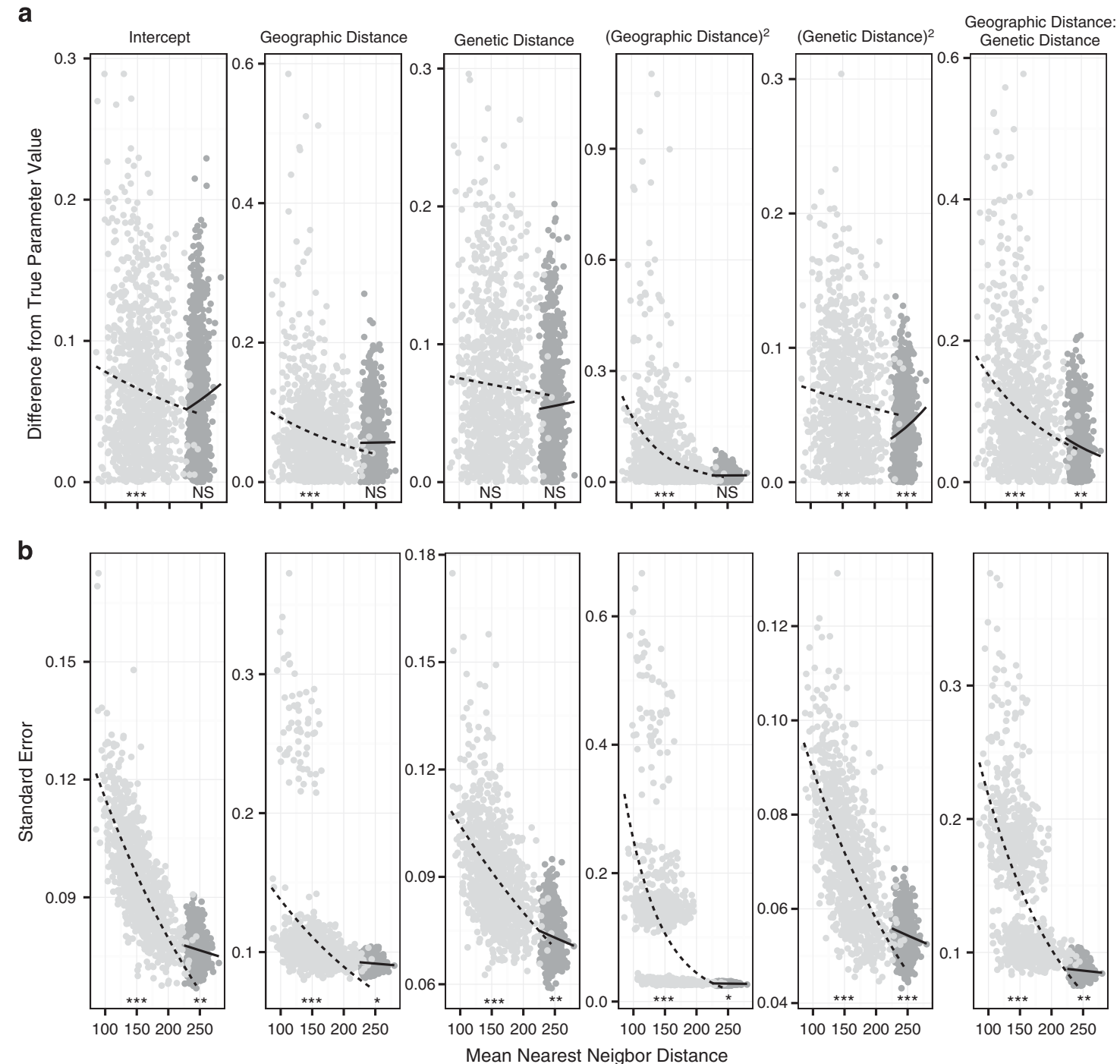

Figure 4 Relationship between measures of lack of model fit and the MNND of those crossing-sets for both SRS (light gray dots and dashed lines) and SPREAD-generated (dark gray dots and solid lines) crossing-sets. Trend lines were calculated using a generalized linear model with a Gaussian response distribution and a log link function. Significance of the line slope is shown below each line: ${ }^{* * *} P \leqslant 0.0001,{ }^{* *} P \leqslant 0.001,{ }^{*} P \leqslant 0.01$, NS $P>0.05$. (a) The absolute value of the difference between population parameter values and sample parameter values of SRS- and SPREADgenerated crossing-sets for all six model parameters. (b) The standard errors of SRS- and SPREAD-generated crossing-sets for all six model parameters. 
original population should be carefully considered because these crosses will often be selected by SPREAD.

Calculating the true MMNND by computing MNND values for every possible subset of a sampled population may not be possible. Instead, our algorithm generates a large number $(h)$ of random crossing-sets and chooses the set with the MMNND from those $h$ crossing-sets. Choosing an appropriate $h$ value is an important consideration when implementing the algorithm because too small an $h$ value may reduce the probability of selecting a crossing-set with an MNND value close to the maximum value, whereas too large an $h$ value would unnecessarily increase computation time.

Our results show that the range of the distribution of MMNND values decreases as $h$ value increases, mainly driven by an increase in the minimum MMNND value. Increasing the $h$ value used in the algorithm increases the probability of obtaining an MMNND value close to the true MMNND value. However, the probability that an additional sample will yield a value higher than all samples already considered decreases as the number of samples already considered goes up. In terms of the number of random samples already sampled (h), this probability can be calculated as $1-\left(\frac{h-1}{h+1}\right)$, for example, in our test, we used $h$ values of $10,100,1000$ and 10000 . The probability of choosing an additional sample with a MNND value outside the range of the samples already taken would be 0.19, 0.0199, 0.001998 and 0.00019998 , respectively. Increasing the $h$ value above 1000 will result in a greater MMNND only $0.2 \%$ of the time. On the basis of this reasoning and our analyses of different $h$ values, using an $h$ value of 1000 should be sufficient for most experiments with modest population sizes.

In experiments with a large breeding stock and small desired experimental mating population, there may be large variation in MNND values among crossing-sets. Such large variation may offset the ability of even large $h$ values to return a crossing-set that is substantially different from a randomly sampled crossing-set. In these cases, SPREAD could be modified to include a simulated annealing function that searches the space of a very large number of potential crossing-sets for a crossing-set that converges on a peak MNND value. One example of a simulated annealing algorithm that could be adapted for this purpose is SAGE (Simulated Annealing Guided Evaluation), developed to design combinatorial drug libraries (Zheng et al., 1999).

We hypothesized that maximizing the diversity inherent in a crossing-set would increase the predictive ability of models relating outcomes to characteristics of crosses. When we compared model fits from crossing-sets generated by SPREAD with model fits from crossing-sets generated by SRS, we found that the model parameter values from SPREAD-generated crossing-sets were more precise (smaller variance) and accurate (smaller mean squared error) estimators of the population parameter values (Table 1 and Figure 3 ).

There was no clear distinction between the bias of parameter values from SPREAD- or SRS-generated crossing-sets. Both methods resulted in sample parameter values that were not equal to the true parameter values, and both methods produced the same number of more or less biased parameter values (Table 1). Because both methods are equally biased, the source of the bias is probably systemic. The population parameter values are from a model calculated using the entire set of 24 mat-A $\times 24$ mat-a crosses while the sample parameter values are from crossing-sets of 12 mat- $\mathrm{A} \times 12$ mat-a strains. The smaller sample size used to fit the model decreases the ability of sample parameter values to estimate population parameter values. Generalized linear models have been shown to be especially sensitive to sample size, compared with other methods (Wisz et al., 2008).
The true MMNND does not need to be determined to produce crossing-sets that provide accurate estimates of underlying population level parameter values. For most parameters, SPREAD-generated crossing-sets with large MNND values fall at the bottom of the curve describing the relationship between the MNND of SRS-generated crossing-sets and either their deviation from population parameter values (Figure $4 \mathrm{a}$ ), or the standard error of sample parameters (Figure $4 \mathrm{~b}$ ). Within the SPREAD-generated crossing-sets (dark gray, Figure 4), the benefit of a slightly higher MNND value to measures of model fit is negligible and, therefore, so is the benefit of increasing the $h$ value used in SPREAD. Furthermore, the negative trend between the MNND of crossing-sets and our two measures of lack of model fit provides strong statistical support for our claim that maximizing the MNND of crossing-sets increases the utility of data generated from those crossing-sets.

Although our analysis used a dataset generated from the fungus Neurospora crassa, SPREAD can be easily used for many other organisms. SPREAD can be used to design breeding experiments for any fungus, plant, animal or other sexual eukaryote with two mating types or sexes where ecological, genetic or physiological trait differences between the individuals involved in a cross are measured. The algorithm can be used directly, and does not require any modification when used with other organisms that satisfy these criteria.

For a species with more than two mating types, for example, the social amoebae Dictyostelium discoideum that has three mating types (I, II and III) (Bloomfield et al., 2010), SPREAD would have to be run for each possible combinations of compatible mating types. The desired crossing-set size would have a maximum size dependent on the mating type with the fewest strains. For $D$. discoideum, SPREAD would have to be run six times for the following combinations: $\mathrm{I} \times(\mathrm{II}, \mathrm{III}) ; \mathrm{II} \times(\mathrm{I}, \mathrm{III}) ; \mathrm{III} \times(\mathrm{I}, \mathrm{II}) ; \mathrm{I} \times \mathrm{II} ; \mathrm{I} \times \mathrm{III} ; \mathrm{II} \times \mathrm{III}$. The crossing-set with the greatest MNND value from the six SPREAD selected crossing-sets would then be used for experimentation. The number of SPREAD runs required will scale rapidly with the number of mating types involved, requiring parallel computation of SPREAD for species with large numbers of mating types.

SPREAD increases the value of fully crossed mating designs by enabling exploration and prediction across the full space of cross characteristics provided by available breeding stock. Simulations based on crossing-sets generated from the SPREAD algorithm vs SRS prove our algorithm generates more precise and accurate parameter estimates, enabling better predictions of relationships between cross characteristics (for example, the genetic and geographic distances between parents) and the success of a cross. SPREAD is not computationally intense and is easy to implement, making it a valuable tool for researchers designing crossing experiments.

\section{DATA ARCHIVING}

$N$. crassa genetic and geographic distance dataset, simulated experimental data, and the data and $\mathrm{R}$ code for figures and tables are available at the Dryad Digital Repository (http://dx.doi.org/10.5061/ dryad.8br20). The $\mathrm{R}$ code for SPREAD is available in the Supplementary Information (Supplementary File 2) and online (http://dx.doi.org/10.6084/m9.figshare.1180165).

\section{CONFLICT OF INTEREST}

The authors declare no conflict of interest.

\section{ACKNOWLEDGEMENTS}

We thank Kareem Carr and Steven Worthington for assistance in designing statistical analyses. We are grateful to members of the Pringle lab for advice and 
discussion. Our work is supported by the National Science Foundation Graduate Research Fellowship under Grant Nos. (DGE0644491 and DGE1144152) awarded to K.Z. and by other National Science Foundation grants awarded to the Pringle Laboratory. This work was also supported by funds from the Max Planck Institute for Demographic Research to KZ, DL and AP. Some computations in this paper were run on the Odyssey cluster supported by the FAS Division of Science, Research Computing Group at Harvard University. The work of DL was supported by the Max-Planck Odense Center, a collaboration of the Max Planck Society and the University of Southern Denmark.

Baddeley A, Turner R (2005). Spatstat: An R package for analyzing spatial point patterns. J Stat Softw 12: 1-42.

Bloomfield G, Skelton J, Ivens A, Tanaka Y, Kay RR (2010). Sex determination in the social amoeba Dictyostelium discoideum. Science 330: 1533-1536.

Clark PJ, Evans FC (1954). Distance to Nearest Neighbor as a Measure of Spatial Relationships in Populations. Ecology 35: 445-453.

Crepieux S, Lebreton C, Servin B, Charmet G (2004). Quantitative trait loci (QTL) detection in multicross inbred designs: recovering QTL identical-by-descent status information from marker data. Genetics 168: 1737-1749.

Cushman SA (2014). Grand challenges in evolutionary and population genetics: the importance of integrating epigenetics, genomics, modeling, and experimentation. Front Genet 5: 1-5.

Ellison CE, Hall C, Kowbel D, Welch J, Brem RB, Glass NL et al. (2011). Population genomics and local adaptation in wild isolates of a model microbial eukaryote. Proc Nat Acad Sci USA 108: 2831-2836.

Fournier DA, Skaug HJ, Ancheta J, lanelli J, Magnusson A, Maunder MN et al. (2012). AD Model Builder: using automatic differentiation for statistical inference of highly parameterized complex nonlinear models. Optim Methods Softw 27: 233-249.

Griffing B (1956). Concept of general and specific combining ability in relation to diallel crossing systems. Aust J Biol Sci 9: 463-493.
Holliday JD, Ranade SS, Willett P (1995). A fast algorithm for selecting sets of dissimilar molecules from large chemical databases. Quantitative Structure-Activity Relationships 14: $501-506$

Jui PY, Lefkovitch LP (1992). Selecting the size of a diallel cross experiment. Theor Appl Genet 85: 21-25.

King JR, Jackson DA (1999). Variable selection in large environmental data sets using principal components analysis. Environmetrics 10: 67-77.

Lynch M (1991). The genetic interpretation of inbreeding depression and outbreeding depression. Evolution 45: 622-629.

Martin EJ, Critchlow RE (1999). Beyond mere diversity: tailoring combinatorial libraries for drug discovery. J Comb Chem 1: 32-45.

Pauli F, Coles S (2001). Penalized likelihood inference in extreme value analyses. J Appl Stat 28: 547-560.

R Core Team (2014). R: A language and Environment for Statistical Computing. R Foundation for Statistical Computing: Vienna, Austria. Available from http://www. R-project.org/.

Skaug H, Fournier D, Nielsen A, Magnusson A, Bolker B (2013). Generalized Linear Mixed Models using AD Model Builder.

Wang X, Cumming SG (2011). Measuring landscape configuration with normalized metrics. Landscape Ecol 26: 723-736.

Weston S (2014) Revolution Analytics. Foreach: Foreach looping construct for R. Wickham H (2007). Reshaping data with the reshape package. J Stat Softw 21: $1-20$.

Wickham H (2009). ggplot2: elegant graphics for data analysis. Springer: New York. Wickham H (2011). The split-apply-combine strategy for data analysis. J Stat Softw 40: $1-29$.

Wisz MS, Hijmans RJ, Li J, Peterson AT, Graham CH, Guisan A et al. (2008). Effects of sample size on the performance of species distribution models. Divers Distrib 14 763-773.

Zheng W, Cho SJ, Waller CL, Tropsha A (1999). Rational combinatorial library design. 3. Simulated annealing guided evaluation (SAGE) of molecular diversity: a novel computational tool for universal library design and database mining. J Chem Inf Model 39: 738-746.

Zhu J, Weir BS (1996). Diallel analysis for sex-linked and maternal effects. Theor Appl Genet 92: 1-9.

Supplementary Information accompanies this paper on Heredity website (http://www.nature.com/hdy) 